\begin{tabular}{|c|c|c|c|c|}
\hline 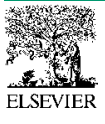 & Review & TRENDS in Cognitive Sciences & Vol.8 No.3 March 2004 & $\begin{array}{l}\text { Full text provided by www.sciencedirect.com } \\
\text { science }(d \text { DirEct. }\end{array}$ \\
\hline
\end{tabular}

\title{
Developmental cognitive neuroscience: progress and potential
}

\author{
Yuko Munakata ${ }^{1}$, B.J. Casey ${ }^{2}$ and Adele Diamond ${ }^{3}$ \\ ${ }^{1}$ Department of Psychology, 345 UCB, University of Colorado, Boulder, Boulder, CO 80309-0345, USA \\ ${ }^{2}$ Sackler Institute for Developmental Psychobiology, Weill Medical College of Cornell University, NY 10021, USA \\ ${ }^{3}$ Center for Developmental Cognitive Neuroscience, University of Massachusetts Medical School, Shriver Center Campus, Waltham, \\ MA 02452, USA
}

\begin{abstract}
Developmental cognitive neuroscience is an evolving field that investigates the relations between neural and cognitive development. Lying at the intersection of diverse disciplines, work in this area promises to shed light on classic developmental questions, mechanisms subserving developmental change, diagnosis and treatment of developmental disorders, and cognitive and neuroscientific topics traditionally considered outside the domain of development. Fundamental questions include: What are the interrelations between developmental changes in the brain (e.g. in connectivity, chemistry, morphology) and developmental changes in children's behavior and cognitive abilities (e.g. representational complexity, ability to sustain selective attention, speed of processing)? Why, and how, is learning enhanced during certain periods in development? How is our knowledge organized, and how does this change with development? We discuss preliminary investigations of such questions and directions for future work.
\end{abstract}

Developmental cognitive neuroscience is an evolving field that investigates the relations between brain development and cognitive development. It has the potential to inform understanding of the mechanisms that subserve perception, attention, memory, language and other cognitive processes at different points in the life cycle, and mechanisms subserving developmental changes in those processes. This field draws upon many others, such as disciplines within psychology, neuroscience, cognitive science, genetics and social science (see Box 1 for the relation between developmental cognitive neuroscience and developmental neuroscience). Work in this area uses methods from all of the related disciplines, including behavioral studies, neuroimaging, molecular genetics, computational modeling, single-cell recording and neurochemical assays. An emphasis is placed on complementary methods - evaluating multiple aspects or levels of developmental processes (from molecular to systems levels), in typical and atypical development, in humans and other species.

Developmental cognitive neuroscience research can speak to classic developmental issues, such as nature versus nurture and continuity versus discontinuity in development. For example, everyone now agrees that the answer to the nature-nurture debate includes some of each (see Box 2 for changes in terminology with advances in the study of development). The real question is how genetic and environmental factors interact during the course of development to shape the brain, mind and behavior. Developmental cognitive neuroscience research can help to address this question. For example, such research has demonstrated how specific genes can affect the pruning and maturation of synapses, which in turn affects the ability to learn from experience [1], and how experience can affect which genes are turned on, when, and how they are expressed (e.g. [2]). Computational models have demonstrated how small variations in initial processing, which might be genetically governed, can lead through experience to large differences in cognitive outcomes [3,4].

By investigating both typical and atypical development, developmental cognitive neuroscience research can inform a variety of practical applications, such as earlier diagnosis and more effective treatment of developmental disorders. For example, such research has informed the

\section{Box 1. Developmental neuroscience}

Whereas developmental cognitive neuroscience is squarely at the intersection of brain development and cognitive development, the field of developmental neuroscience pertains to the physical development of the brain, the cellular and molecular events that underlie nervous system development [74]. The two fields also differ in that developmental neuroscientists have traditionally focused primarily on embryonic and very early postnatal development.

Many developmental neuroscientists study mechanisms that direct the molding of the anatomical and functional organization of brain, such as axonal guidance, synapse formation and the development of neuronal connectivity. Intensive analyses are conducted, for example, of the cellular and molecular mechanisms (including signal transduction pathways) that stimulate, inhibit, regulate or perturb axonal and dendritic outgrowth, neuronal and glial migration, and establishment of ordered neural maps.

A central problem in developmental neuroscience is to identify the signaling molecules (such as neurotrophic factors and cytokines) that control and arrest cell differentiation and migration. Another central problem is the expression and function of regulatory genes (which control such aspects of brain development as neuronal morphology and excitability, or the expression of neurotransmitters and their receptors) and regionalization of gene transcription. 


\section{Box 2. Terminology in the study of development}

Early terminology focused on hard-and-fast contrasts in development (such as nature vs. nurture, genes vs. environment, and maturation vs. learning) and hard-and-fast time windows (such as critical periods):

Learning: changes in response to experience with the environment (the nurture side of nature-nurture, when this experience comes from a caretaker)

Maturation: changes driven by genetic processes according to specific timetables (the nature side of nature-nurture)

Critical periods: time-limited windows when specific experiences must occur to drive typical or maximal development. Learning is ineffective outside these time windows.

However, it became evident that such hard-and-fast distinctions are too simplistic, and this is acknowledged in later terminology (such as experience-expectant and experience-dependent processes, which incorporate genetic and environmental factors):

Experience-expectant processes [33]: processes that utilize environmental information that is highly reliable for all members of the species (e.g. for humans, hearing a language)

Experience-dependent processes [33]: processes that utilize environmental information that can vary across individuals (e.g. for humans, the particular language that is heard)

Sensitive periods: time-limited windows when specific experiences have their largest effects. Learning can still be effective outside those time windows.

In addition, more neutral terminology refers to general aspects of change:

Plasticity: the capacity for modification

Development: processes of change across the lifespan

treatment for the genetic disorder, phenylketonuria (PKU). Individuals with PKU cannot convert phenylalanine to tyrosine, the precursor to the neurotransmitter dopamine. Treatment involves restricting dietary intake of phenylalanine, thus allowing ingested tyrosine more opportunity to compete with phenylalanine for transport into the brain. Developmental cognitive neuroscience research showed that the recommended diet had not restricted phenylalanine intake enough and resulted in deficits in executive control functions, but that a stricter diet could prevent and reverse those deficits $[5,6]$. Further, if the gross elevations in blood phenylalanine levels are not reduced before Postnatal Day 11, they impair the visual system, which develops rapidly after birth, and deficits in sensitivity to low contrast are still evident many years later [7], similar to the long-lasting deficits seen after neonatal cataracts corrected in the first month [8].

As another example, schizophrenia is a devastating neuropsychiatric disorder with an onset usually in young adulthood. Animal research has indicated that neonatal perturbation of the hippocampus disrupts normal prefrontal cortex development and its regulation by dopaminergic activity [9,10]. Behavioral effects are not seen immediately, but are evident years later in heightened reactivity to stress and poorer executive control, which are characteristic of schizophrenia. Similar perturbation of the hippocampus in adulthood does not produce such effects. Not only might this provide insight into schizophrenia etiology, it also provides insight into the complex interactions that are important for normal development. This is but one example of how studying atypical development can inform understanding of typical development.

Developmental cognitive neuroscience work can also inform issues that have traditionally been considered outside the domain of development. For example, to what extent is adult cognition subserved by domain-specific systems that are intrinsically specialized for particular kinds of inputs (e.g. a face processing system [11]), as opposed to domain-general systems that become shaped for particular kinds of inputs through learning [12]? Both possibilities are compatible with newborn preferences to look towards face-like patterns, which probably reflect subcortical processes rather than the cortical specializations debated in adults [13]. Developmental cognitive neuroscience research could inform these debates [14] by evaluating whether domain-specific systems appear early in development, before much learning has occurred (supporting the intrinsically specialized view), or only later with the development of expertise (supporting the domain-general view).

In the selective review that follows, we discuss several specific questions of investigation in the field and assess preliminary answers (for additional discussion and coverage of a broad range of topics in developmental cognitive neuroscience, see [15-20]).

\section{Neural changes during learning}

How does brain organization and function change during the process of learning, and how does this compare with changes observed across development? The increasing availability of non-invasive tools, such as functional magnetic resonance imaging (fMRI, [21,22]), provides us with the opportunity to ask such brain- and behaviorrelated questions in the developing human that was not possible only a decade ago. With fMRI, we can safely track changes in cortical activation following extensive learning in the same individual, and we can compare such changes with those observed in younger versus older children.

One of the first studies to track cortical changes over an extensive period of time with fMRI [23] showed rapid learning effects in primary motor areas. Changes were shown during motor sequence learning within a single session and increased over weeks of training. Cortical activity became less diffuse and increased over time. This example of initial diffuse cortical activity early in learning, followed by an increase in focal activity, parallels results from developmental fMRI studies. These studies show diffuse activity in children relative to adolescents and adults, with adolescents showing the greatest focal activity during performance of behavioral tasks, even when performance across groups is equated [24-29].

Differences in brain activity between age groups are not due to experience alone, as even without normal stimulation, changes in neuronal connections and synaptic pruning occur with brain maturation [30]. Rather, these findings highlight a possible approach for investigating maturational and experiential contributions. For example, to determine whether the immature brain after extended practice engages in the same neural processes as the mature one, we could compare brain activity in the mature system with brain activity in the immature system both 
before and following extended experience. This use of fMRI to trace learning-related changes in cortical areas is currently being used to investigate the impact of behavioral and cognitive interventions on developmental disorders like dyslexia and obsessive-compulsive disorder.

Neuroanatomical changes also occur with learning and development, notably changes in the strength and number of neuronal connections and the myelination of fibers. During early development, the neural connections in the brain undergo dramatic organization, generating more neuronal processes and connections than will ultimately survive (e.g. [31,32]). Learning plays a key role in an activity-mediated competition process through which some of these synapses are eliminated or pruned, and others are stabilized and strengthened [1,33,34].

Developmental studies have challenged some accepted notions about neural organization and learning. It has long been known that monocular deprivation causes changes in ocular dominance columns in primary visual cortex (V1), but it had been assumed that the effects of visual experience were passed along from the eye to the thalamus (the lateral geniculate) and from there to V1. However, recent work has shown that physiological changes occur more rapidly in V1 than in the thalamus [35]. Moreover, protein synthesis in V1 is necessary for rapid plasticity; anatomical changes in thalamo-cortical afferents are not [36]. This work suggests that cortical circuitry is probably the substrate of the rapid plasticity in response to visual experience (or the lack thereof), whereas thalamo-cortical changes might then make those changes hard to reverse.

\section{Learning across development}

Why is learning sometimes enhanced during certain periods in development? That is, why do there appear to be 'sensitive periods' in development, during which learning is most effective? For example, the ability to learn the grammar of a language declines with age [37,38]. Sensitive periods for other kinds of learning fall within the first few years or months of life, for example, for the phonemes of one's mother tongue [39] and for certain aspects of face processing [40].

Computational models, specifically neural network simulations, have been used to investigate potential mechanisms for such sensitive periods (e.g. [41,42]). These models allow researchers complete control over simulated learning systems and their environments, to help identify factors contributing to enhanced learning during particular points in development.

One set of simulations [43] investigated the possibility that sensitive periods in language learning arise from the advantages of 'starting small' (see also [38]). Specifically, less-developed working memory abilities might facilitate language learning, by restricting attention to key elements of language input, highlighting the grammatical structure of the language. Simulations testing this idea demonstrated that limitations in working memory could in fact lead to advantages in language processing. Other simulations have demonstrated that such advantages to starting small might not be present under all circumstances [44], and that networks have a tendency to start small on their own, abstracting basic structure before abstracting more complex structure, even without limitations imposed on their processing [45]. In this way, computational models have proven to be a useful tool for investigating how and when starting small might assist in learning.

Neural network simulations have also been used to investigate the possibility that sensitive periods arise because knowledge becomes entrenched as a system learns, making it more difficult to alter the system with subsequent learning. Such simulations have demonstrated that entrenchment can occur as some units and connections become committed and unused connections are pruned [41,42]. The result is that stimuli encountered early are learned more robustly than stimuli encountered later.

Entrenchment can also occur as a result of counterproductive learning, where attempts to learn new information actually lead to the strengthening of incorrect responses. Simulations have shown how such processes can explain sensitive periods in phoneme discrimination [46]. Specifically, an infant (or network) might start with an auditory perceptual system that responds differentially to sounds in the environment, and strengthens these responses with experience. For example, a learner in a Japanese language environment will experience a sound that is a blend of the English / $\mathrm{r} /$ and / / sounds. This sound will activate relevant neurons, and learning will lead to a strengthening of their response. When later faced with an English / $\mathrm{r} /$ or $/ \mathrm{l} /$, the existing representation of the single blended sound will be activated in this system. Learning will again tend to strengthen this response, but in this case the learning will be counterproductive, strengthening the tendency to hear the English /r/ and /// sounds as the single blended sound. The interaction of these learning processes and environmental inputs can thus lead to sensitive periods for learning phonemes.

\section{Organization and development of knowledge}

Just as it is clear that nature versus nurture is a false dichotomy, so it is clear that cognition, emotion, perception and motor functions are not nearly as separate as previously thought. They are fundamentally, multiply intertwined. For example, which variant a child has of the COMT gene (the gene that codes for the COMT enzyme, important for clearing dopamine from extracellular space in prefrontal cortex [47]) can affect that child's cognitive functioning [48] and reactivity to stress [49]. Analyses of genes, such as the COMT gene, that affect neurotransmitter systems, provide a window into neurochemical modulation of cognitive and affective function.

Another example involves the interaction of social and affective systems with perceptual and attentional systems. Aberrant social experience (i.e. early physical abuse) appears to affect children's perceptual processing and discrimination abilities (e.g. [50,51]). Children with and without histories of severe physical abuse identify happiness, sadness and fear equally well, but physically abused children are expert at recognizing subtle signs of anger. They have a much lower perceptual threshold for seeing faces as angry, both at the behavioral and neural levels. 
They also require greater attentional and neural resources than other children to disengage their attention from an angry face to focus on a target.

Interrelations between motor and cognitive processing provide another example where systems are more intertwined than previously thought. Fine motor skills and visual-motor coordination mature together with higher cognitive functions, neither reaching full maturity until late adolescence [52,53]. Attention-deficit hyperactivity disorder (ADHD) is a developmental disorder that affects not only cognitive functioning [54,55], but the motivational system [56,57] and motor functioning [58]. Just as ADHD and developmental coordination disorder are overlapping diagnoses in many children, movement deficits are evident in many children with 'cognitive' developmental disorders (such as dyslexia, specific language disorder, and autism; $[59,60])$. In addition, children who have undergone resection of cerebellar tumors (without cranial irradiation or chemotherapy) show not only motor deficits, but also impairments in executive cognitive functions, such as planning, sequencing and working memory, and deficits in affect modulation $[61,62]$. Pre-supplementary motor cortex and part of premotor cortex are active during motor and mental (numerical, verbal, and spatial) sequential operations [63].

Mirror neurons provide another example. Originally discovered in monkeys [64], these neurons fire when an individual performs a particular action or observes another individual performing that action. Such mirror neurons are found in humans in roughly the same location as in monkeys (premotor cortex and Area 44 in prefrontal cortex). They fire during imitation or observation of gestures [65] and during imitation or observation of emotional expression [66]. Such neurons provide a mechanism for integrating perception and action at the neuronal level. Mirror neurons might contribute to several developmental processes, such as the imitative behavior of infants (which requires the integration of perceived and performed actions [67]), the correlation between infants' abilities to perform actions and to comprehend those actions in others [68], and the development of empathy (understanding how another feels because you can identify with that feeling in yourself [64]).

\section{Future directions}

Progress in the field of developmental cognitive neuroscience has been rapid, but much potential remains to be tapped (see also Box 3 for some areas where caution is needed). We close with a brief consideration of issues that we view as central to the endeavor of understanding relations between brain and cognitive development.

\section{Interactions and integrations}

Future work should, and probably will, continue to move away from dichotomies (e.g. nature vs. nurture, domainspecific vs. domain-general systems, and cognition vs. emotion) and from studying aspects of the developing system in isolation. Future work is likely to focus on specifying the complex interactions among various processes (including interactions among different neurotransmitters, and among chemical, physiological and structural

\section{Box 3. Cautions about the application of developmental cognitive neuroscience research}

Given the rapid pace of advances and discoveries in the field of developmental cognitive neuroscience, there is an understandable level of excitement and attention regarding work in this area. Research in this field has great potential for informing how caregivers raise children, how educators teach students, and how clinicians diagnose and treat patients. We believe the excitement surrounding this field, and the potential applications of this research, increase the need for caution in interpreting study results and their implications. Much work remains to be done, and changes to public policy based on developmental cognitive neuroscience research could in some cases be premature.

For example, findings from developmental cognitive neuroscience have focused attention, particularly in the United States, on the importance of children's experiences during the first three years of life (see discussion in [75]). As discussed in this article, there are rapid brain changes during this period such as synaptic exuberance and pruning, and it is a sensitive period for effective learning in a variety of domains. In addition, the complexity of the environment has been shown to influence synapse formation (e.g. [76]). These strands of evidence have been put together to argue that the first three years of life are a critical period, during which caregivers must work to provide abundant stimulation for children or face the lifelong consequences of suboptimal development. However, that conclusion is almost certainly inaccurate (e.g. [75]). For example, the period of rapid synapse formation early in life does not define the time window for learning. Critical periods for learning are complex, graded and variable, and not simply open and then closed. Effects of the environment on the brain can be observed throughout life, and the mature adult brain shows striking plasticity, even neurogenesis $[77,78]$. Such findings do not imply constant levels of plasticity across the lifespan, but do suggest that appropriate caution is needed in drawing links between neural changes and critical periods. Moreover, although evidence is strong that brain development is better in a more species-typical environment than in an impoverished environment with little stimulation, social interaction, or room for activity or exploration, the evidence is sparse and unclear on the effects of trying to create a supra-optimal environment. Thus, developmental cognitive neuroscience research might ultimately speak to the kinds and timing of experiences that are most effective for development, but current evidence certainly does not dictate a protocol that caregivers must follow during a 'critical period' of children's development.

Remediation is another area where developmental cognitive neuroscience research has great potential, but some caution is advised. For example, such research has led to the development of programs for reading remediation (e.g. [79]), which have yielded improvements in performance. However, interpreting such results requires control treatments that are equated with the remediation treatments in all ways except those thought to be essential for remediation. Such controls are often lacking, making it difficult to assess the effectiveness of the remediation programs [80].

changes in the brain), and how they affect, and are affected by, behavior and psychological development (see also Box 4). For example, the role of hormones and stress should be considered within a full picture of the relation between brain development and cognitive development [69].

Another area concerns the interaction of genetic and environmental factors during development. In addition to the factors contributing to sensitive periods discussed earlier, the timing of biological events is clearly important. For example, one type of post-synaptic receptor molecule, the NMDA receptor, changes during development in a way that could contribute to learning being easier when we are 


\section{Box 4. Questions for future research}

- What are the most promising areas for unified developmental cognitive neuroscience accounts that can explain behavior in terms of specific developmental interactions among genes, neuroanatomy, neurochemistry, neurophysiology, and the environment (social, cultural, and physical)?

- How are the neural underpinnings of cognition, emotion, perception and motor development interrelated?

- How can developmental cognitive neuroscience research inform the development of better teaching strategies and remediation programs for language, mathematics, and other skills?

- How can developmental cognitive neuroscience research aid in the early detection and treatment of developmental disorders?

- What aspects of brain and cognitive development are common across species, and what factors contribute to developments unique to the human species?

younger [70]. Although this might appear to be a maturational process under genetic control, the change in the NMDA receptor is experience-dependent, and can be reversed if experience is withheld [71]. Understanding such interplay between genes and environment is likely to be crucial for understanding sensitive periods, and development more generally.

\section{Complementarity}

The use of complementary methods will become increasingly important in developmental cognitive neuroscience, as new tools are developed and as thinking regarding the relations among multiple levels of developmental processes advances. For example, combining fMRI with electroencephalography (EEG) allows researchers to take advantage of the spatial resolution of fMRI for identifying precisely where in the brain activity changes are occurring, and the temporal resolution of EEG for capturing neural changes linked to rapidly occurring cognitive changes [72].

Such complementary methods are informative in cognitive neuroscience in general, but could be particularly fruitful in the study of development. For example, neuroimaging methods might allow infants and young children to be tested with relatively simple tasks or with no task at all (where stimuli are simply presented for viewing). This would support testing children across a wide range of ages in the same experiment, thus advancing understanding of developmental changes [14]. In addition, altered cognitive processing might be revealed earlier through neuroimaging than in behavior, because compensatory cognitive and behavioral strategies can mask problems. This is particularly relevant in development, for example allowing teachers, parents and doctors to detect problems earlier and so intervene earlier and more successfully.

Diffusion tensor imaging (DTI) is a relatively new imaging tool, providing a non-invasive means for assessing brain connectivity [73]. The human brain undergoes extensive postnatal development in neuronal connectivity, as axons, dendrites and synapses are formed or enlarged and others are pruned, and the strength of this connectivity is modulated as neural tracts undergo an on-going process of myelination. In combination with fMRI and
EEG, DTI might ultimately provide us with the opportunity to relate changes in connectivity and myelination to behavioral and neurophysiological measures of changes in the speed and efficiency of cognitive and neural processing during development. From a basic neuroscience perspective, it provides the unique ability to determine in vivo both function and connectivity within developing and mature brains. Such complementary approaches will be essential for informing our understanding of how the brain and behavior change with development.

\section{Conclusion}

The field of developmental cognitive neuroscience is advancing rapidly, along with the many fields that it draws upon. Developmental cognitive neuroscience research promises to provide a rich understanding of the interrelations among developmental changes in cognitive, perceptual, emotion and motor processing and in the brain's anatomy, chemistry and physiology, and the interaction of genetic and environmental factors in driving such development. This kind of understanding should inform the study of classic developmental and cognitive issues, and may in some cases redefine the form such questions take.

\section{Acknowledgements}

The writing of this review was supported by the James S. McDonnell Foundation Bridging Brain, Mind, and Behavior Program (JSMF Grant\#21002016, 21st Century Science Collaborative Activity Award).

\section{References}

1 Churchill, J.D. et al. (2002) A converging-methods approach to fragile X syndrome. Dev. Psychobiol. 40, 323-338

2 Cancedda, L. et al. (2003) Patterned vision causes CRE-mediated gene expression in the visual cortex through PKA and ERK. J. Neurosci. 23, 7012-7020

3 Oliver, A. et al. (2000) Deviations in the emergence of representations: a neuroconstructivist framework for analysing developmental disorders. Dev. Sci. 3, 1-23

4 O'Reilly, R.C. and Johnson, M.H. (2002) Object recognition and sensitive periods: a computational analysis of visual imprinting. In Brain Development and Cognition: a Reader (Johnson, M.H. et al., eds), pp. 392-413, Blackwell

5 Diamond, A. (2001) A model system for studying the role of dopamine in prefrontal cortex during early development in humans. In Handbook of Developmental Cognitive Neuroscience (Nelson, C. and Luciana, M., eds), pp. 433-472, MIT Press

6 Luciana, M. et al. (2001) Associations between phenylalanine-totyrosine ratios and performance on tests of neuropsychological function in adolescents treated early and continuously for phenylketonuria. Child Dev. 72, 1637-1652

7 Diamond, A. and Herzberg, C. (1996) Impaired sensitivity to visual contrast in children treated early and continuously for PKU. Brain 119, 101-116

8 Maurer, D. and Lewis, T.L. (2001) Visual acuity: the role of visual input in inducing postnatal change. Clin. Neurosci. Res. 1, 239-247

9 Bertolino, A. et al. (2002) Reduced N-acetylaspartate in prefrontal cortex of adult rats with neonatal hippocampal damage. Cereb. Cortex $12,983-990$

10 Lipska, B.K. and Weinberger, D.R. (2002) A neurodevelopmental model of schizophrenia: neonatal disconnection of the hippocampus. Neurotox. Res. 4, 469-475

11 Kanwisher, N. (2000) Domain specificity in face perception. Nat. Neurosci. 3, 759-763

12 Tarr, M.J. and Gauthier, I. (2000) FFA: a flexible fusiform area for subordinate-level visual processing automatized by expertise. Nat. Neurosci. 3, 764-770

13 de Haan, M. et al. (2002) Specialization of neural mechanisms 
underlying face recognition in human infants. J. Cogn. Neurosci. 14, 199-209

14 Spelke, E.S. (2002) Developmental neuroimaging: a developmental psychologist looks ahead. Dev. Sci. 5, 392-396

15 Casey, B.J., de Haan, M. eds (2002) Special Issue on 'New Methods in Developmental Science' Blackwell

16 Casey, B.J., Munakata, Y. eds (2002) Special Issue on 'Converging Methods Approach to Developmental Science' Wiley

17 Diamond, A. et al. Developmental Cognitive Neuroscience, Oxford University Press (in press)

18 Johnson, M.H. (2001) Functional brain development in humans. Nat. Rev. Neurosci. 2, 475-483

19 Johnson, M.H. (1997) Developmental Cognitive Neuroscience: an Introduction, Blackwell Publishers

20 Nelson, C.A., Luciana, M. eds (2001) Handbook of Developmental Cognitive Neuroscience, MIT Press

21 Kwong, K.K. et al. (1992) Dynamic magnetic resonance imaging of human brain activity during primary sensory stimulation. Proc. Natl. Acad. Sci. U. S. A. 89, 5675-5679

22 Ogawa, S. et al. (1990) Oxygenation-sensitive contrast in magnetic resonance image of rodent brain at high magnetic fields. Magn. Reson. Med. 14, 68-78

23 Karni, A. et al. (1995) Functional MRI evidence for adult motor cortex plasticity during motor skill learning. Nature 377, 155-158

24 Casey, B.J. et al. (1997) A developmental functional MRI study of prefrontal activation during performance of a go-nogo task. J. Cogn. Neurosci. 9, 835-847

25 Casey, B.J. et al. (2002) Dissociating striatal and hippocampal function developmentally with a stimulus-response compatibility task. J. Neurosci. 22, 8647-8652

26 Hertz-Pannier, L. et al. (1997) Noninvasive assesment of language dominance in children and adolescents with functional MRI: a preliminary study. Neurology 48, 1003-1012

27 Klingberg, T. et al. (2001) Increased brain activity in frontal and parietal cortex underlies the development of visuospatial working memory capacity during childhood. J. Cogn. Neurosci. 14, 1-10

28 Luna, B. et al. (2001) Maturation of widely distributed brain function subserves cognitive development. Neuroimage 13, 786-793

29 Schlaggar, B. et al. (2002) Functional neuroanatomical differences between adults and school-age children in the processing of single words. Science 296, 1476-1479

30 Bourgeois, J.P. et al. (1994) Synaptogenesis in the prefrontal cortex of rhesus monkeys. Cereb. Cortex 4, 78-96

31 LaMantia, A.S. and Rakic, P. (1994) Axon overproduction and elimination in the anterior commissure of the developing rhesus monkey. J. Comp. Neurol. 340, 328-336

32 Huttenlocher, P.R. and Dabholkar, A.S. (1997) Regional differences in synaptogenesis in human cerebral cortex. J. Comp. Neurol. 387, 167-178

33 Greenough, W. et al. (1987) Experience and brain development. Child Dev. 58, 539-559

34 Goodman, C.S. and Shatz, C.J. (1993) Developmental mechanisms that generate precise patterns of neuronal connectivity. Cell 72 (Suppl), 77-98

35 Trachtenberg, J.T. and Stryker, M.P. (2001) Rapid anatomical plasticity of horizontal connections in the developing visual cortex. J. Neurosci. 21, 3476-3482

36 Taha, S. and Stryker, M.P. (2002) Rapid ocular dominance plasticity requires cortical, but not geniculate, protein synthesis. Neuron 34 , $425-436$

37 Hakuta, K. et al. (2003) A test of the critical-period hypothesis for second-language acquisition. Psychol. Sci. 14, 31-38

38 Newport, E.L. (1990) Maturational constraints on language learning. Cogn. Sci. 14, 11-28

39 Kuhl, P.K. et al. (1992) Linguistic experience alters phonetic perception in infants by 6 months of age. Science 255, 606-608

40 Geldart, S. et al. (2002) The effects of early visual deprivation on the development of face processing. Dev. Sci. 5, 490-501

41 Ellis, A.W. and Lambon-Ralph, M.A. (2000) Age of acquisition effects in adult lexical processing reflect loss of plasticity in maturing systems: insights from connectionist networks. J. Exp. Psychol. Learn. Mem. Cogn. 26, 1103-1123
42 Zevin, J. and Seidenberg, M.S. (2002) Age of acquisition effects in reading and other tasks. J. Mem. Lang. 47, 1-29

43 Elman, J.L. (1993) Learning and development in neural networks: the importance of starting small. Cognition 48, 71-99

44 Rohde, D.L.T. and Plaut, D.C. (1999) Language acquisition in the absence of explicit negative evidence: how important is starting small. Cognition 72, 67-109

45 Munakata, Y. and McClelland, J.L. (2003) Connectionist models of development. Dev. Sci. 6, 413-429

46 McClelland, J.L. et al. (1999) Understanding failures of learning: Hebbian learning, competition for representational space, and some preliminary experimental data. In Brain, Behavioral, and Cognitive Disorders: the Neurocomputational Perspective (Reggia, J. et al., eds), pp. 75-80, Elsevier

47 Sesack, S.R. et al. (1998) Dopamine axon varicosities in the prelimbic division of the rat prefrontal cortex exhibit sparse immunoreactivity for the dopamine transporter. J. Neurosci. 18, 2697-2708

48 Diamond, A. et al. Genetic and neurochemical modulation of prefrontal cognitive functions in children. Am. J. Psychiatry (in press)

49 Zubieta, J. et al. (2003) COMT val158met genotype affects mu-opioid neurotransmitter responses to a pain stressor. Science 299, 1240-1243

50 Pollack, S.D. and Kistler, D.J. (2002) Early experience is associated with the development of categorical representations for facial expressions of emotion. Proc. Natl. Acad. Sci. U. S. A. 99, 9072-9076

51 Pollack, S.D. and Tolley-Schell, S.A. (2003) Selective attention to facial emotion in physically abused children. J. Abnorm. Psychol. 112, $323-338$

52 Diamond, A. (2000) Close interrelation of motor development and cognitive development and of the cerebellum and prefrontal cortex. Child Dev. 71, 44-56

53 Rosenbaum, D.A. et al. (2001) Skills of mind and skills of eye, ear, and muscle are fundamentally similar. Annu. Rev. Psychol. 52, 453-470

54 Barkley, R.A. et al. (1992) Frontal lobe functions in attention deficit disorder with and without hyperactivity: a review and research report. J. Abnorm. Child Psychol. 20, 163-188

55 Pennington, B.F. et al. (1993) Contrasting cognitive deficits in attention deficit hyperactivity disorder versus reading disability. Dev. Psychol. 29, 511-523

56 Crone, E.A. et al. (2003) Sensitivity to interference and response contingencies in attention-deficit/hyperactivity disorder. J. Child Psychol. Psychiatry 44, 214-226

57 Sergeant, J. (2000) The cognitive-energetic model: an empirical approach to attention-deficit hyperactivity disorder. Neurosci. Biobehav. Rev. 24, 7-12

58 Gillberg, C. (2003) Deficits in attention, motor control, and perception: a brief review. Arch. Dis. Child. 88, 904-910

59 Hardan, A.Y. et al. (2003) Motor performance and anatomic magnetic resonance imaging (MRI) of the basal ganglia in autism. J. Child Neurol. 18, 317-324

60 Noterdaeme, M. et al. (2002) Evaluation of neuromotor deficits in children with autism and children with a specific speech and language disorder. Eur. Child Adolesc. Psychiatry 11, 219-225

61 Levisohn, L. et al. (2000) Neurospychological consequences of cerebellar tumor resection in children. Brain 123, 1041-1050

62 Riva, D. and Giorgi, C. (2000) The cerebellum contributes to higher functions during development: evidence from a series of children surgically treated for posterior fossa tumours. Brain 123, 1051-1061

63 Hanakawa, T. et al. (2002) The role of rostral Brodmann area 6 in mental-operation tasks: an integrative neuroimaging approach. Cereb. Cortex $12,1157-1170$

64 Rizzolatti, G. et al. (1996) Premotor cortex and the recognition of motor actions. Brain Res. Cogn. Brain Res. 3, 131-141

65 Grezes, J. et al. (2003) Activations related to 'mirror' and 'canonical' neurons in the human brain: an fMRI study. Neuroimage 18, 928-937

66 Carr, L. et al. (2003) Neural mechanisms of empathy in humans: a relay from neural systems for imitation to limbic areas. Proc. Natl. Acad. Sci. U. S. A. 100, 5497-5502

67 Meltzoff, A.N. and Decety, J. (2003) What imitation tells us about social cognition: a rapprochement between developmental psychology and cognitive neuroscience. Philos. Trans. R. Soc. Lond. B Biol. Sci. 358, 491-500

68 Woodward, A.L. and Guajardo, J.J. (2002) Infants' understanding of the point gesture as an object-directed action. Cogn. Dev. 83, 1-24 
69 McEwen, B.S. (1997) Homrones as regulators of brain development: life-long effects related to health and disease. Acta Paediatr. Suppl. 422, 41-44

70 Carmignoto, G. and Vicini, S. (1992) Activity-dependent decrease in NMDA receptor responses during development of the visual cortex. Science 258, 1007-1011

71 Quinlan, E.M. et al. (1999) Bidirectional, experience-dependent regulation of $\mathrm{N}$-methyl-D-aspartate receptor subunit composition in the rat visual cortex during postnatal development. Neurobiology 96 , 12876-12880

72 van der Meij, W. et al. (2001) The existence of two sources in rolandic epilepsy: confirmation with high resolution EEG, MEG and fMRI. Brain Topogr. 13, 275-282

73 Pierpaoli, C. and Basser, P.J. (1996) Toward a quantitative assessment of diffusion anisotropy. Magn. Reson. Med. 36, 893-906

74 Jessell, T.M. and Sanes, J.R. (2000) The decade of the developing brain. Curr. Opin. Neurobiol. 10, 599-611

75 Bruer, J.T. (1999) The Myth of the First Three Years: A New
Understanding of Early Brain Development and Lifelong Learning, The Free Press, New York

76 Greenough, W.T. et al. (1985) Evidence for active synapse formation and altered post-synaptic metabolism in the visual cortex of rats reared in complex environments. Proc. Natl. Acad. Sci. U. S. A. 82 4549-4552

77 Gould, E. et al. (1999) Neurogenesis in the neocortex of adult primates. Science 286, 548-552

78 Kempermann, G. (2002) Regulation of adult hippocampal neurogenesis: implications for novel theories of major depression. Bipolar Disord. 4, 17-33

79 Tallal, P. et al. (1996) Language comprehension in language-learning impaired children improved with acoustically modified speech. Science 271, 81-84

80 Olson, R. and Wise, B. Computer programs for reading remediation and controlled comparisons of methods. In Handbook of Literacy and Technology (Vol. 2) (McKenna, M. et al., eds), Lawrence Erlbaum (in press)

\section{News \& Features on BioMedNet}

Start your day with BioMedNet's own daily science news, features, research update articles and special reports. Every two weeks, enjoy BioMedNet Magazine, which contains free articles from Trends, Current Opinion, Cell and Current Biology. Plus, subscribe to Conference Reporter to get daily reports direct from major life science meetings.

\section{http://news.bmn.com}

Here is what you will find in News \& Features:

\section{Today's News}

Daily news and features for life scientists.

Sign up to receive weekly email alerts at http://news.bmn.com/alerts

\section{Special Report}

Special in-depth report on events of current importance in the world of the life sciences.

\section{Research Update}

Brief commentary on the latest hot papers from across the Life Sciences, written by laboratory researchers chosen by the editors of the Trends and Current Opinions journals, and a panel of key experts in their fields.

Sign up to receive Research Update email alerts on your chosen subject at http://update.bmn.com/alerts

\section{BioMedNet Magazine}

BioMedNet Magazine offers free articles from Trends, Current Opinion, Cell and BioMedNet News, with a focus on issues of general scientific interest. From the latest book reviews to the most current Special Report, BioMedNet Magazine features Opinions, Forum pieces, Conference Reporter, Historical Perspectives, Science and Society pieces and much more in an easily accessible format. It also provides exciting reviews, news and features, and primary research. BioMedNet Magazine is published every 2 weeks. Sign up to receive weekly email alerts at http://news.bmn.com/alerts

\section{Conference Reporter}

BioMedNet's expert science journalists cover dozens of sessions at major conferences, providing a quick but comprehensive report of what you might have missed. Far more informative than an ordinary conference overview, Conference Reporter's easy-to-read summaries are updated daily throughout the meeting.

Sign up to receive email alerts at http://news.bmn.com/alerts 